\title{
Bladder tumor recurrence after urothelial carcinoma of the upper urinary tract
}

\author{
Peter E. Lonergan, Sima P. Porten \\ Department of Urology, Helen Diller Family Comprehensive Cancer Center, University of California, San Francisco, California, USA \\ Contributions: (I) Conception and design: SP Porten; (II) Administrative support: None; (III) Provision of study materials or patients: None; \\ (IV) Collection and assembly of data: All authors; (V) Data analysis and interpretation: All authors; (VI) Manuscript writing: All authors; (VII) Final \\ approval of manuscript: All authors. \\ Correspondence to: Sima P. Porten, MD, MPH. Department of Urology, Helen Diller Family Comprehensive Cancer Center, University of California, \\ San Francisco, 550 16th Street, San Francisco, CA 94143, USA. Email: Sima.Porten@ucsf.edu.
}

\begin{abstract}
Upper tract urothelial carcinoma (UTUC) is a relatively uncommon and poorly investigated malignancy, however, bladder recurrence after radical nephroureterectomy (RNU) is a frequent event. In this review, we summarize the current knowledge on risk prediction of bladder tumor recurrence after RNU, including surgical strategies and adjuvant intravesical treatments to reduce the risk of recurrence. Finally, we outline some of the more recent advances in genomics that will likely lead to new prognostic markers and risk stratification tools that may refine UTUC treatment in the future.
\end{abstract}

Keywords: Upper tract urothelial carcinoma (UTUC); intravesical recurrence; nephroureterectomy; surveillance

Submitted Feb 23, 2020. Accepted for publication Mar 24, 2020.

doi: $10.21037 /$ tau.2020.03.47

View this article at: http://dx.doi.org/10.21037/tau.2020.03.47

\section{Incidence \& epidemiology}

Urothelial carcinomas are the 5 th most frequently diagnosed non-cutaneous cancer in the US (1) and may be located in the lower genitourinary tract (urethra and bladder) or upper genitourinary tract (ureter and pyelocaliceal system). Upper tract urothelial carcinoma (UTUC) is a relatively rare entity, accounting for $5-7 \%$ of all renal tumors and $5-10 \%$ of all urothelial tumors. The estimated annual incidence is 1-2 cases per 100,000 (1,2). Recent advances in imaging and endoscopic techniques, as well as improved bladder cancer survival has led to an increase in the detection of UTUC. The mean age at diagnosis has also increased over the last 30 years from 68 to 73 years (3). The cause of UTUC is still unknown, however several environmental factors, such as cigarette smoking, herbal medicines (e.g., aristolochic acid), chronic infection, and occupational carcinogenesis, have been linked to the development of UTUC (4). Due to the low prevalence of the disease, racial differences in the development of UTUC is inconclusive. Asian ethnicity appears to be associated with higher-grade disease compared with other ethnicities (5). However, stage for stage, there appears to be no racial differences. Differences in epigenetic and genetic factors such as occupation/environmental exposures as well as socioeconomic factors may explain these differences.

The standard of care for surgical management of UTUC is radical nephroureterectomy (RNU) with excision of bladder cuff (6). Intravesical recurrence occurs in approximately $50 \%$ of patients during follow-up surveillance with ureteric tumors and high tumor grade independent predictors of a bladder recurrence (7). The majority of these recurrences occur in the first year after surgery (8).

\section{Risk prediction}

UTUC and bladder cancer have common pathological mechanisms and show analogous tumor characteristics with similar prognostic risk factors $(9,10)$. As a result, clinical decision-making for UTUC is often inferred from the larger evidence base on primary bladder cancer (11). However, UTUC remains an aggressive disease with high 
progression and recurrence rates. Conversely, certain patients with lower risk disease may benefit from a more conservative approach, such as segmental resection or endoscopic ablation. Pre-operative risk stratification with individualized risk prediction of bladder recurrences after RNU may inform the frequency of surveillance and identify patients who are most likely to benefit from adjuvant intravesical treatments. A recent meta-analysis identified three categories of predictors of intravesical recurrence after RNU: (I) patient-specific factors including preexisting renal impairment, male gender, smoking and a prior history of bladder cancer; (II) tumor-specific factors including multifocality, location with the ureter, positive preoperative cytology, $\geq \mathrm{pT} 2$ and histological evidence of necrosis; (III) treatment-specific factors including positive surgical margins, extravesical bladder cuff excision and a laparoscopic approach (12). However, it is difficult to draw robust conclusions from many of these studies as they are limited by their small sample size, single-center series, heterogeneous cohorts and multivariable risk models which do not adequately integrate surgery and tumor specific variables.

Two multivariable models for predicting intravesical recurrences after RNU are currently available. Xylinas and colleagues from the UTUC Collaboration Group (13) developed and validated a risk stratification nomogram predicting bladder recurrences at 3, 6, 9, 12, 18 and 36 months. In a cohort of 1,839 patients managed with RNU at 15 European and North American centers over a 20year period, $31 \%$ of patients had a bladder recurrence at a median of 24 months. In multivariable models, advanced age, male gender, ureteral tumor location, laparoscopic surgical technique, endoscopic distal ureteral management, previous bladder cancer, higher tumor stage, concomitant carcinoma in situ (CIS), and lymph node involvement were all associated with intravesical recurrence. The authors proposed two versions of the nomogram-a full model and a reduced model, with discriminative accuracies of $69.0 \%$ and $67.8 \%$ respectively in the validation cohort. The reduced model was based exclusively on previously described clinicopathologic features of patients with UTUC, whereas the full model incorporated the surgical approach (e.g., open versus laparoscopic approach) and endoscopic management or stripping of distal ureter versus transvesical approach.

Another nomogram has also been developed in a cohort of 754 patients in 13 institutions in Japan (14). In this study, the incidence of intravesical recurrence at 12 months was
$15 \%$ and $29 \%$ at 5 years with a median time to recurrence of 10 months. Multivariate analysis found that papillary tumor architecture, absence of lymphovascular invasion and higher pathological T stage ( $\geq$ pT2) were independently significant predictive factors and that male gender was a marginally significant predictive factor for intravesical recurrence. Age, laterality, body mass index, smoking status, tumor location, multiplicity, tumor grade, concomitant CIS, hydronephrosis, positive preoperative urine cytology, management of distal ureter, surgical approach and adjuvant chemotherapy were not associated with recurrence in the bladder.

Both studies have inherent limitations which should be acknowledged. Centralized pathologic review was not performed in either study and may have led to misinterpretation of pathologic specimens and underreporting of certain features such as CIS. Neither study controlled for smoking status or cumulative exposure, which this has been previously shown to be associated with bladder recurrences after RNU (13). Evolving surgical technique related to the long time periods in both studies were not accounted for and may have profound effects on the outcomes. The effect of the laparoscopic approach on risk of bladder recurrence still remains unclear.

While these studies hold promise for predicting intravesical recurrences after RNU and raise important questions regarding appropriate follow up endoscopic surveillance and adjuvant treatment, they must be rigorously externally validated before they can be introduced into routine clinical practice.

\section{Strategies to reduce the risk of bladder recurrence}

Strategies to reduce to the risk of intravesical recurrence after RNU for UTUC fall under two broad categories: (I) surgical technique and (II) intravesical treatments. Open RNU with bladder cuff excision has historically been the standard-of-care for high risk UTUC, regardless of tumor location (6). Initially described by Clayman and colleagues (15) almost 30 years ago, laparoscopic RNU has been widely adopted in the last decade. Numerous techniques to manage the bladder cuff during laparoscopic RNU have also been described: via an open approach (intravesical or extravesical excision), a laparoscopic approach (standard excision, laparoscopic stapling, laparoscopic bipolar sealer/divider instrument), or via an endoscopic approach (transurethral resection with ureter stripping). A robot-assisted laparoscopic approach has been recently 
described in order to facilitate management of the distal ureter (16). Most published series suggest better perioperative outcomes using a minimally invasive approach (17), with favorable oncologic outcomes. A recent European Association of Urology (EAU) Guidelines systematic review of 42 studies of 7,554 patients comparing the oncologic outcomes of laparoscopic compared with open RNU found that all but 1 of the included studies were retrospective series, and most reported similar oncologic outcomes between laparoscopic and open RNU (18). Only 26 of the included studies reported bladder recurrence-free survival and most studies reported equivalent or better bladder recurrence-free survival with a laparoscopic approach (18). These results should be interpreted with caution as most of the studies were small-sample and underpowered to detect a difference in oncologic efficacy between surgical approaches. Only 9 of the 41 retrospective series adjusted for confounders, therefore their findings are difficult to interpret with the selection bias favoring the laparoscopic group in most studies. Finally, a meta-analysis of the data was not possible due to the underlying heterogeneity of the studies (18).

Several precautions have been advocated by the EAU in order to reduce the risk of tumor recurrence: (I) avoid entering the urinary tract; (II) avoid direct contact between instruments and the tumor; (III) laparoscopic RNU must take place in a closed system including avoiding morcellation of the tumor and the use an endobag for tumor extraction; (IV) removal of the kidney and ureter en bloc with the bladder cuff; (V) invasive or large (T3/T4 and/or $\left.\mathrm{N}_{+} / \mathrm{M}_{+}\right)$tumors are possible contraindications for laparoscopic RNU $(6,18)$.

Early ureteral ligation has also been proposed as a means of preventing seeding of upper urinary tract cells to the bladder as a cause of intravesical recurrence. This has been evaluated in a single-arm prospective trial in 74 patients undergoing RNU and compared with a propensity scorematched historical control cohort. Twenty-three percent of patients who had early ureteral ligation during RNU developed a bladder recurrence with a median followup of 24 months. Although there was no difference in the intravesical recurrence-free survival rates in patients with ureteral disease, a significant difference was observed in patients with UTUC of the renal pelvis. Multivariate analyses also identified early ureteral ligation as an independent predictor of intravesical recurrence in patients with UTUC located in the renal pelvis (19).

With intravesical recurrence rates up to $50 \%$ after RNU there has been considerable interest in adjuvant bladder installations to reduce the risk of bladder recurrence. Two prospective randomized trials $(20,21)$, a meta-analysis (22) and a Cochrane systematic review (23) have shown that a single post-operative instillation of intravesical chemotherapy [mitomycin C (MMC), pirarubicin (THP)] in the early post-operative period reduces the risk of bladder tumor recurrence within the first year following RNU.

The prospective ODMIT-C (One Dose Mitomycin C) Trial was undertaken in 46 UK centers in 284 patients with no previous or concurrent history of bladder cancer undergoing RNU for suspected UTUC. Forty mg MMC was administered on removal of the urinary catheter. Intravesical recurrence was assessed by visual inspection at cystoscopy at 3, 6, and 12 months after RNU. Overall, a single dose of post-operative intravesical MMC led to an absolute reduction in risk of intravesical recurrence of $11 \%$ and the number needed to treat to prevent one bladder tumor was nine (20). The principle limitations of the trial were the lack of histological confirmation of recurrence and also the lack of standardized timing of the administration of MMC which occurred at least a week after RNU. Although the trial was not designed to investigate the efficacy of surgical approaches for RNU, 29 of 284 patients (10\%) underwent laparoscopic RNU, and a recurrence within the bladder was only found in $3(10 \%)$ patients. At the time the trial was opened, laparoscopic urologic surgery was in its infancy and the low level of recurrence may be due to case selection bias favoring smaller tumors for laparoscopic approaches.

The efficacy of a single intravesical instillation of $30 \mathrm{mg}$ THP within 48 hours after RNU has also been evaluated in a prospective trial in 77 patients from 11 institutions in Japan (21). Follow-up cystoscopy and urinary cytology were repeated every 3 months for 2 years or until the first recurrence. Overall, the investigators found that fewer patients who received THP had a recurrence compared to the control group (16.9\% at 1 year and $16.9 \%$ at 2 years in the THP group compared with $31.8 \%$ at 1 year and $42.2 \%$ at 2 years in the control group; log-rank $\mathrm{P}=0.025)$. On multivariable analysis THP instillation [hazard rate (HR), 0.26; 95\% CI, 0.07-0.91; $\mathrm{P}=0.035$ ] and open surgery (HR, 0.28 ; $95 \%$ CI, $0.09-0.84 ; \mathrm{P}=0.024)$ were independently predictive of a decreased incidence of intravesical recurrence. In this study, histologic confirmation of the bladder recurrence was required. Multivariable analysis did find that laparoscopic surgery was associated with an increased risk of intravesical recurrence. As with the previous trial, this study was not designed to evaluate the 
surgical approach for managing UTUC. Future trials are needed to assess the head-to-head comparisons of chemotherapeutic drugs, as well as determine the optimal timing of chemotherapy instillations.

\section{Surveillance and treatment of bladder recurrences}

With the high risk of intravesical recurrences, it is mandatory for patients with UTUC to undergo endoscopic surveillance after RNU. Surveillance schedules consist of cystoscopy and urinary cytology for at least 5 years regardless of the stage or grade of primary UTUC (6), however there is little evidence to guide the frequency of surveillance or risk-adapted strategies for follow-up. The two available multivariable models for predicting intravesical recurrences after RNU do not recommend specific cut offs for determining recurrence risk in the context of endoscopic surveillance and these thresholds cannot be easily derived from the decision curve analysis in either study $(13,14)$.

Recent data using conditional survival analysis suggest that the risk of intravesical recurrence evolves during surveillance after RNU. The concept of conditional survival probability shows the likelihood of surviving for an additional duration after the initial diagnosis or disease treatment because the patient has already survived for a certain time. It provides a more accurate and dynamic estimate of outcome probability at each surveillance follow-up point and has been recently assessed in UTUC $(24,25)$. Conditional intravesical recurrence-free survival has been reported in 364 patients with Ta-3N0M0 disease who underwent either open or laparoscopic RNU (24). Bladder recurrences were detected in 176 (48.4\%) patients and based on 1, 2, 3 and 4-year survivorship the 5 -year conditional intravesical recurrence-free survival rate increased from $41.5 \%$ to $60.5 \%, 73.4 \%, 79.5 \%$ and $96.7 \%$, respectively. Predictive factors of bladder recurrences including laparoscopic RNU and pT stage 2 or less remained independent risk factors over time and bladder recurrences typically developed within 18 months, particularly 6 to 12 months after RNU. As a result, patients with pT2 or lower T stage who undergo laparoscopic RNU may be recommended for longer follow-up (24). The study was limited by its small sample size and retrospective nature, as well as a lack of information regarding established prognostic factors such as smoking status.

The management of a bladder recurrence following
RNU is similar to the current guideline-based treatment strategy for primary bladder cancer, however, there is a lack of data addressing the natural history of intravesical recurrences and progression in patients with UTUC after treatment with RNU. Tanaka and colleagues reviewed the records of 241 patients with an intravesical recurrence after RNU (pTa-4N0M0) (26). All patients were treated with transurethral resection of the bladder recurrence and 101 (41.9\%) underwent Bacillus Calmette-Guerin (BCG) treatment, whereas 49 (20.3\%) underwent intravesical chemotherapy. Multivariate analysis found that $\mathrm{pT} 1$ disease and the number of tumor recurrences at the time of first bladder recurrence were independent risk factors for a recurrence. Disease progression within the bladder was associated with pT1 tumors, concomitant CIS and no prior BCG treatment (26).

\section{Genomic advances}

UTUC is histologically similar to urothelial bladder cancer however genomic studies have shown they are distinct entities (27). Although UTUC shares many of the same genomic alterations with urothelial carcinoma of the bladder, some key differences have been identified as oncogenic drivers of UTUC (28). As a result, there has been considerable interest in elucidating genomic alterations in UTUC which could lead to novel prognostic markers that may refine UTUC treatment and post-treatment surveillance.

One particular recent study has offered a new insight into the genomic differences between UTUC and bladder cancer by defining the clonal relatedness of temporally distinct tumors (29). The investigators prospectively sequenced tumors and matched germline DNA in a cohort of 195 UTUC patients and 454 primary bladder cancer patients using a targeted next-generation sequencing platform. Although the spectrum of genomic alterations was similar between UTUC and primary bladder cancer, significant differences in the prevalence of mutations in individual genes were found, including a higher frequency of FGFR3 and $H R A S$ alterations and a lower frequency of TP53, RB1, and $E R B B 2$ alterations in UTUC as compared with bladder cancer. Of the 195 patients with UTUC, 137 underwent RNU, with 57 (42\%) later developing an intravesical recurrence. In a subgroup of 29 patients with UTUC and a history of intravesical recurrence, both tumors were analyzed to assess their clonal relatedness and were found to be consistently clonally related. After adjusting for clinical 
factors associated with intravesical recurrences, alterations in FGFR3, KDM6A and CCND1 were associated with a high risk of developing a subsequent bladder recurrence, whereas TP53 alterations were associated with a lower risk (29). This result is of particular importance as it confirms that UTUC with subsequent bladder recurrence are clonally related, justifying developing methods to prevent lower tract seeding during RNU and the identification of patients with genetic alterations that may require more rigorous surveillance after surgery.

\section{Conclusions}

In summary, although UTUC is a relatively rare entity, bladder recurrence after RNU is a relatively frequent event. In recent years, considerable advances have been made in developing prognostic tools to identify patients at a higher risk of intravesical recurrence who may benefit from intensified therapy and those who could be spared from the side effects of unnecessary intervention and follow up surveillance. In the future it is likely that routine genomic characterization of UTUC will provide more clinically relevant information, including identification of patients who could be candidates for molecularly driven clinical trials or for the evaluation of the risk of bladder recurrence, which may help guide the selection of adjuvant treatment and intensity of follow-up surveillance.

\section{Acknowledgments}

Funding: None.

\section{Footnote}

Provenance and Peer Review: This article was commissioned by the Guest Editors (Jay D. Raman and John Knoedler) for the series "Upper-Tract Urothelial Carcinoma: current state and future directions" published in Translational Andrology and Urology. The article was sent for external peer review organized by the Guest Editors and the editorial office.

Conflicts of Interest: Both authors have completed the ICMJE uniform disclosure form (available at http://dx.doi. org/10.21037/tau.2020.03.47). The series "Upper-Tract Urothelial Carcinoma: current state and future directions" was commissioned by the editorial office without any funding or sponsorship. The authors have no other conflicts of interest to declare.
Ethical Statement: The authors are accountable for all aspects of the work in ensuring that questions related to the accuracy or integrity of any part of the work are appropriately investigated and resolved.

Open Access Statement: This is an Open Access article distributed in accordance with the Creative Commons Attribution-NonCommercial-NoDerivs 4.0 International License (CC BY-NC-ND 4.0), which permits the noncommercial replication and distribution of the article with the strict proviso that no changes or edits are made and the original work is properly cited (including links to both the formal publication through the relevant DOI and the license). See: https://creativecommons.org/licenses/by-nc-nd/4.0/.

\section{References}

1. Siegel RL, Miller KD, Jemal A. Cancer statistics, 2020. CA Cancer J Clin 2020;70:7-30.

2. Soria F, Shariat SF, Lerner SP, et al. Epidemiology, diagnosis, preoperative evaluation and prognostic assessment of upper-tract urothelial carcinoma (UTUC). World J Urol 2017;35:379-87.

3. Raman JD, Messer J, Sielatycki JA, et al. Incidence and survival of patients with carcinoma of the ureter and renal pelvis in the USA, 1973-2005. BJU Int 2011;107:1059-64.

4. Colin P, Koenig P, Ouzzane A, et al. Environmental factors involved in carcinogenesis of urothelial cell carcinomas of the upper urinary tract. BJU Int 2009;104:1436-40.

5. Matsumoto K, Novara G, Gupta A, et al. Racial differences in the outcome of patients with urothelial carcinoma of the upper urinary tract: an international study. BJU Int 2011;108:E304-9.

6. Roupret M, Babjuk M, Comperat E, et al. European Association of Urology Guidelines on Upper Urinary Tract Urothelial Carcinoma: 2017 Update. Eur Urol 2018;73:111-22.

7. Zigeuner RE, Hutterer G, Chromecki T, et al. Bladder tumour development after urothelial carcinoma of the upper urinary tract is related to primary tumour location. BJU Int 2006;98:1181-6.

8. Raman JD, Ng CK, Boorjian SA, et al. Bladder cancer after managing upper urinary tract transitional cell carcinoma: predictive factors and pathology. BJU Int 2005;96:1031-5.

9. Novara G, De Marco V, Gottardo F, et al. Independent predictors of cancer-specific survival in transitional cell carcinoma of the upper urinary tract: multiinstitutional dataset from 3 European centers. Cancer 
2007;110:1715-22.

10. Sylvester RJ, van der Meijden AP, Oosterlinck W, et al. Predicting recurrence and progression in individual patients with stage Ta T1 bladder cancer using EORTC risk tables: a combined analysis of 2596 patients from seven EORTC trials. Eur Urol 2006;49:466-75; discussion 475-7.

11. Kim M, Jeong CW, Kwak C, et al. Are urothelial carcinomas of the upper urinary tract a distinct entity from urothelial carcinomas of the urinary bladder? Behavior of urothelial carcinoma after radical surgery with respect to anatomical location: a case control study. BMC Cancer 2015;15:149.

12. Seisen T, Granger B, Colin P, et al. A Systematic Review and Meta-analysis of Clinicopathologic Factors Linked to Intravesical Recurrence After Radical Nephroureterectomy to Treat Upper Tract Urothelial Carcinoma. Eur Urol 2015;67:1122-33.

13. Xylinas E, Kluth L, Passoni N, et al. Prediction of intravesical recurrence after radical nephroureterectomy: development of a clinical decision-making tool. Eur Urol 2014;65:650-8.

14. Ishioka J, Saito K, Kijima T, et al. Risk stratification for bladder recurrence of upper urinary tract urothelial carcinoma after radical nephroureterectomy. BJU Int 2015;115:705-12.

15. Clayman RV, Kavoussi LR, Figenshau RS, et al. Laparoscopic nephroureterectomy: initial clinical case report. J Laparoendosc Surg 1991;1:343-9.

16. Brandao LF, Autorino R, Zargar H, et al. Robot-assisted laparoscopic adrenalectomy: step-by-step technique and comparative outcomes. Eur Urol 2014;66:898-905.

17. Hanna N, Sun M, Trinh QD, et al. Propensity-scorematched comparison of perioperative outcomes between open and laparoscopic nephroureterectomy: a national series. Eur Urol 2012;61:715-21.

18. Peyronnet B, Seisen T, Dominguez-Escrig JL, et al. Oncological Outcomes of Laparoscopic Nephroureterectomy Versus Open Radical Nephroureterectomy for Upper Tract Urothelial Carcinoma: An European Association of Urology Guidelines Systematic Review. Eur Urol Focus 2019;5:205-23.

19. Yamashita S, Ito A, Mitsuzuka K, et al. Efficacy of early ureteral ligation on prevention of intravesical recurrence after radical nephroureterectomy for upper urinary tract urothelial carcinoma: a prospective single-arm multicenter clinical trial. Jpn J Clin Oncol 2017;47:870-5.
20. O'Brien T, Ray E, Singh R, et al. Prevention of bladder tumours after nephroureterectomy for primary upper urinary tract urothelial carcinoma: a prospective, multicentre, randomised clinical trial of a single postoperative intravesical dose of mitomycin $\mathrm{C}$ (the ODMIT-C Trial). Eur Urol 2011;60:703-10.

21. Ito A, Shintaku I, Satoh M, et al. Prospective randomized phase II trial of a single early intravesical instillation of pirarubicin (THP) in the prevention of bladder recurrence after nephroureterectomy for upper urinary tract urothelial carcinoma: the THP Monotherapy Study Group Trial. J Clin Oncol 2013;31:1422-7.

22. Fang D, Li XS, Xiong GY, et al. Prophylactic intravesical chemotherapy to prevent bladder tumors after nephroureterectomy for primary upper urinary tract urothelial carcinomas: a systematic review and metaanalysis. Urol Int 2013;91:291-6.

23. Hwang EC, Sathianathen NJ, Jung JH, et al. Single-dose intravesical chemotherapy after nephroureterectomy for upper tract urothelial carcinoma. Cochrane Database Syst Rev 2019;5:CD013160.

24. Shigeta K, Kikuchi E, Hagiwara M, et al. The Conditional Survival with Time of Intravesical Recurrence of Upper Tract Urothelial Carcinoma. J Urol 2017;198:1278-85.

25. Ploussard G, Xylinas E, Lotan Y, et al. Conditional survival after radical nephroureterectomy for upper tract carcinoma. Eur Urol 2015;67:803-12.

26. Tanaka N, Kikuchi E, Kanao K, et al. Independent predictors for bladder outcomes after treatment of intravesical recurrence following radical nephroureterectomy in patients with primary upper tract urothelial carcinoma. Ann Surg Oncol 2014;21:3151-8.

27. Green DA, Rink M, Xylinas E, et al. Urothelial carcinoma of the bladder and the upper tract: disparate twins. J Urol 2013;189:1214-21.

28. Kammerer-Jacquet SF, Mathieu R, Peyronnet B, et al. Genomics in upper tract urothelial carcinoma. Curr Opin Urol 2017;27:35-40.

29. Audenet F, Isharwal S, Cha EK, et al. Clonal Relatedness and Mutational Differences between Upper Tract and Bladder Urothelial Carcinoma. Clin Cancer Res 2019;25:967-76.

Cite this article as: Lonergan PE, Porten SP. Bladder tumor recurrence after urothelial carcinoma of the upper urinary tract. Transl Androl Urol 2020;9(4):1891-1896. doi: 10.21037/ tau.2020.03.47 